\title{
La Politique nationale de la ruralité et ses effets sur la santé des communautés rurales du Québec
}

\author{
André-Anne Parent ${ }^{a}$, Paule Simard ${ }^{b}$, Mary Richardson $^{c}, J^{\prime}$ lie Richard ${ }^{\mathrm{d}}$
}

RÉSUMÉ. Cette recherche a pour objectif de mieux comprendre les effets de la Politique nationale de la ruralité (PNR) du Québec sur la santé et le bien-être des individus et des communautés. Elle se base sur une étude de cas multi-site, dans six municipalités réparties dans trois Municipalités régionales de comté du Québec. Une approche qualitative de type interprétative a été retenue, mettant de l'avant une analyse thématique à travers un cadre conceptuel reposant sur les déterminants de la santé. Les résultats démontrent la contribution des projets et processus déployés dans le cadre de la PNR affectant positivement les déterminants de la santé, notamment, la capacité à fournir des services, à encourager les saines habitudes de vie et à favoriser la cohésion sociale. En outre, cette recherche a permis d'illustrer de façon concrète la manière dont la Politique nationale de la ruralité contribue à la santé des individus et des communautés rurales.

\begin{abstract}
The main objective of this research is to gain a better understanding of the effects of Quebec's National Rural Policy on the health and well-being of individuals and communities. The research is based on a multi-site case study, in six municipalities of three regional county municipalities of Quebec. An interpretive qualitative approach was used, as well as a thematic analysis through a conceptual framework based on the determinants of health. The results demonstrate the contribution of projects and processes under the National Rural Policy that positively affect the determinants of health, such as the ability to provide services, encourage bealthy lifestyles, or foster social cohesion. The research has concretely illustrated the ways in which the National Rural Policy contributes to the health of individuals and rural communities.
\end{abstract}

\section{Introduction}

Le monde rural est aujourd'hui marqué par des tensions, où apparaissent d'un côté des facteurs de dynamisme, tels la mise en valeur d'attraits pour le tourisme, l'afflux de nouveaux arrivants et une valorisation de l'immobilier patrimonial, et de l'autre, des facteurs de dévitalisation, comme le vieillissement de la population, l'exode des jeunes, l'étalement urbain, la disparition des services de proximité et un manque de relève dans l'agriculture (OCDE, 2010). Le Québec rural, où vit $26 \%$ de la population, englobe $90 \%$ du territoire habité et comprend 1011 municipalités et 34 communautés autochtones (Desrosiers, 2014; Jean et al., 2014). En 2002, le ministère des Affaires municipales et des régions (MAMR) a mis en place une Politique nationale de la ruralité du Québec (PNR), une initiative relativement rare et innovatrice, puisque peu de pays ont une politique rurale spécifique et les questions de ruralité sont généralement incluses dans les politiques sectorielles (agricole, économique, etc.) (Woods, 2006). Cette politique a connu deux éditions (2002-2007 et 2007-2014). Une troisième édition a été adoptée en 2013 (2014-2021) par le gouvernement en place, mais dès l'arrivée au pouvoir d'un nouveau parti politique en 2014, la PNR a été abrogée et des changements significatifs liés aux instances régionales et locales sont entrés en vigueur, venant ainsi modifier la dynamique de développement des régions ${ }^{1}$.

\footnotetext{
a Professeure adjointe, École de travail social, Université de Montréal

b Chercheure retraitée, Institut national de santé publique du Québec

c Anthropologue-consultante, travailleuse autonome

d Doctorante en santé communautaire, professionnelle de recherche, UQO et UQAR (campus de Lévis)
} 
Très peu d'écrits portent sur les effets des politiques rurales sur la santé des individus et des communautés bien que les déterminants de la santé soient l'objet de recherches depuis de nombreuses années et qu'il existe différents cadres conceptuels pour les étudier (Marmot, 2008; Mikkonen et Raphael, 2010; Raphael, 2016). Au Québec, le ministère de la Santé et des Services sociaux (MSSS) a également développé des cadres et des outils permettant au public et aux chercheurs de comprendre les liens entre la santé et le revenu, la scolarité, le logement, l'emploi, les réseaux de soutien, entre autres (MSSS, 2012). Dans la mesure où ces approches s'intéressent aux liens entre l'organisation et la distribution des ressources économiques et sociales d'une société et la santé des populations, il est fort pertinent de s'attarder aux politiques publiques en tant que moyen à utiliser pour améliorer la santé (Raphael, 2016).

Afin de mieux comprendre les effets des politiques rurales sur la santé, et plus spécifiquement de la PNR, un projet de recherche a été mené de 2013 à 2016 sous la direction de Paule Simard de l'Institut national de santé publique du Québec; cet article rapporte les résultats de cette recherche. Dans cette perspective, l'étude reposait sur la prémisse que les retombées de la PNR peuvent être documentées par l'analyse des projets menés par les agents de développement rural et leur action sur les déterminants de la santé. Pour les besoins de cette étude, les auteurs ont choisi d'utiliser la «Carte de la santé et de ses déterminants » du ministère de la santé et des services sociaux du Québec comme cadre conceptuel et d'analyser les projets et les processus initiés par les agents de développement rural (ADR) (MSSS, 2012). À cet effet, deux stratégies de la PNR ont été étudiées : 1) les pactes ruraux, des ententes signées entre le gouvernement du Québec et les Municipalités Régionales de Comté (MRC) rurales, associées à un financement géré par les MRC pour promouvoir le développement et soutenir le travail en partenariat; 2) l'action des ADR soutenant des communautés dans la réalisation de projets.

Cette recherche visait à : 1) identifier les déterminants sur lesquels deux stratégies de la PNR agissent et repérer les indicateurs permettant de les documenter; 2) décrire et analyser les mécanismes territoriaux (dans les MRC) et locaux (dans les municipalités) par lesquels les Plans d'action des Pactes ruraux et le mandat des ADR sont définis et déployés; 3) mettre en lumière les effets de la PNR sur la santé et le bien-être des individus et des communautés.

\section{Méthodes}

Cette recherche repose sur une étude de cas multi-site, menée dans trois Municipalités régionales de comté (MRC) (MRC d'Arthabaska, du Granit et de Montmagny) et dans deux municipalités par MRC. Elle a été menée, dans une perspective de recherche partenariale (Clément et al., 1995; Bernoux. 2004; Simard, 2005; Darbelley et Paulsen, 2008; Minkler et Wallerstein, 2008; Lincoln, Lynham et Guba, 2018) avec le MAMR, le MSSS, le Réseau québécois des villes et villages en santé et Solidarité rurale du Québec, par une équipe de recherche multidisciplinaire (santé publique, géographie, travail social, anthropologie). Afin d'approfondir la compréhension, interpréter et corroborer les résultats de la recherche, plusieurs méthodes de collecte de données ont été utilisées : 20 entrevues individuelles auprès d'acteurs clés de chaque MRC et des municipalités, 7 entrevues de groupe auprès de 4 à 8 citoyens engagés dans des projets locaux et finalement, 30 entrevues téléphoniques auprès de citoyens non engagés dans un projet de la PNR, ont été réalisées. Ces entretiens avaient pour objectif de documenter les processus mis en place, les mécanismes de gouvernance, et le point de vue des personnes impliquées ou non, dans les projets territoriaux et locaux au sujet des changements qu'ils observent dans leur milieu de vie. Ce projet a reçu l'approbation éthique de la Direction de santé publique de Montréal qui avait à ce moment le mandat d'évaluer les demandes de certification éthique pour l'Institut national de santé publique du Québec.

\subsection{Sélection et descriptions des MRC participantes}

Les critères de sélection des MRC ont été définis de concert avec le MAMR et Solidarité rurale du Québec : variété de types de MRC comprenant à la fois des municipalités ayant un indice de développement négatif et positif; particulièrement actives dans le Pacte rural; ayant une diversité de projets; et situées dans un rayon d'environ $300 \mathrm{~km}$ autour de la ville de Québec. Certains partenaires de la recherche ont suggéré des MRC potentielles qui respectaient ces critères de sélection, à la suite des échanges avec les MRC (préfets ou directeur généraux et ADR) ont permis de recueillir de l'information sur les municipalités en fonction de ces critères. Le choix final des municipalités a été 
fait par les chercheurs. Ce choix a permis de collecter des données qui mettent en lumière des réalités contrastées et qui éclairent sur la diversité des expériences vécues. Les trois MRC retenues offrent un bon éventail de configurations de territoires et de dynamiques en présence.

\subsubsection{Stratégie d'analyse des résultats}

Une analyse thématique du corpus de données a été réalisée (gouvernance du PR, rôle des ADR, projets, retombées, etc.), par MRC et par municipalité (Paillé et Mucchielli, 2012). La synthèse des données a d'abord fait l'objet de trois portraits de milieux, avant de procéder à des analyses transversales sur l'ensemble des milieux. À la figure 1, on trouve une présentation détaillée du cadre conceptuel de l'action sur les déterminants de la PNR.

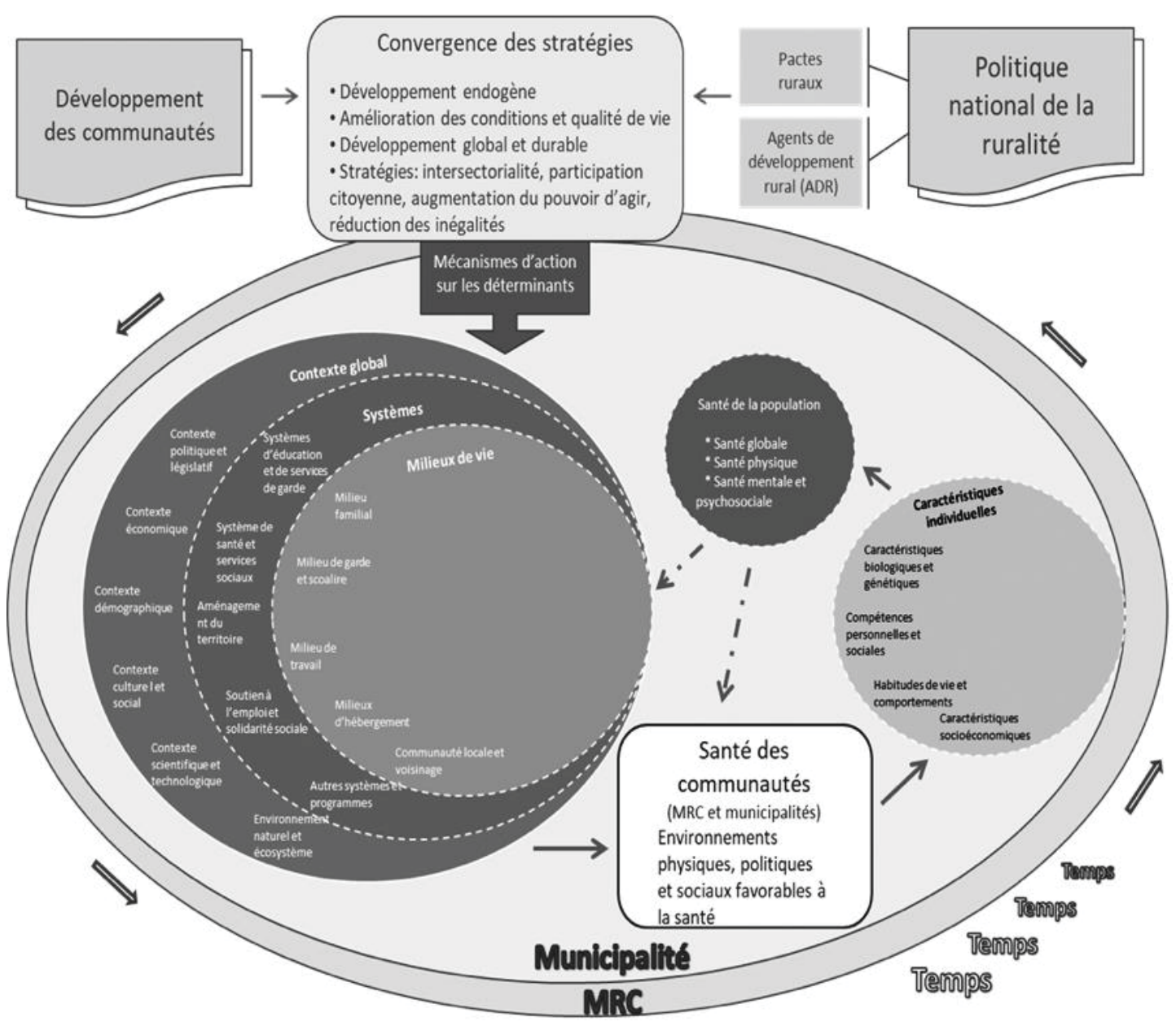

Figure 1 - Cadre conceptuel : Comment la PNR agit-elle sur les déterminants de la santé?

Pour identifier les déterminants touchés par les projets et les processus mis en place dans le cadre de la PNR, les étapes suivantes ont été réalisées : 1) Repérer les retombées de la PNR identifiées par les répondants (MRC et municipalités) en précisant à quelle composante elles référaient (projet ou processus); 2) Mener un premier exercice d'interprétation afin d'illustrer le lien entre les déterminants perçus (c'est-à-dire, tel que nommés par les répondants) et les déterminants de la Carte; 3) Identifier les déterminants de la santé correspondants.

\section{Résultats}

La recherche reposait sur l'idée que la PNR suscite un développement endogène des communautés lo- 
cales, à l'image de la stratégie de soutien au développement des communautés, inscrite dans les orientations de santé publique. La recherche réalisée a permis de constater que l'action sur les déterminants se fait non seulement par le biais de projets (qui peuvent avoir des effets sur le contexte, les milieux de vie, les habitudes et capacités des individus, etc.), mais aussi par les processus initiés au sein des communautés pour répondre à leurs aspirations et leurs besoins. Alors que les projets peuvent fournir des services, encourager de saines habitudes de vie ou favoriser la cohésion sociale, les processus permettent aux membres de la communauté, par exemple, de se donner une vision de leur développement, d'agir sur la rétention de la population, de susciter un sentiment d'appartenance, de créer des collaborations ou de mobiliser la population sur divers aspects qui la concernent. Les résultats sont présentés selon les trois objectifs de la recherche.

\subsection{Objectif 1 : Décrire et analyser les mécanismes territoriaux (MRC) et locaux (municipalités) par lesquels les Plans d'action des Pactes ruraux et le mandat des ADR sont définis et déployés}

L'analyse des mécanismes mis en place à l'échelle de la MRC et des municipalités qui encadrent le déploiement des plans d'action des Pactes ruraux et le mandat des ADR a permis de dégager trois principaux thèmes : 1) les mécanismes de gouvernance mis en place; 2) l'action des agents de développement; 3) les projets collectifs déployés.

\subsubsection{Les mécanismes de gouvernance du Pacte rural}

Les mécanismes de gouvernance sont similaires dans les trois MRC : un comité territorial définit les orientations de gestion des projets, sous l'autorité du Conseil des maires qui entérine ses décisions. Le comité réunit le préfet et le directeur général de la MRC avec quelques représentants des maires et de divers secteurs (communautaire, économique, éducation, etc.). L'étude des projets se fait en plusieurs étapes, à partir d'une grille d'analyse. Les projets locaux émergent parfois de processus collectifs, parfois d'un individu, d'un organisme ou d'un élu. Ces projets doivent nécessairement bénéficier de l'appui du conseil municipal et d'une contribution financière du milieu (caisse populaire, bénévolat, etc.). Diverses mesures sont prises pour ne pas nuire aux milieux qui mettent plus de temps à élaborer un projet dans une démarche participative, notamment l'obligation dans les trois MRC de créer un comité local pour la confection du plan d'action de développement local. Des comités locaux et territoriaux de projets peuvent s'ajouter. Le Pacte rural est perçu par les acteurs des MRC étudiées comme un moyen de soutenir des processus collectifs ou pour aller chercher d'autres fonds ou encore, comme un fonds de dernier recours pour des projets qui ne cadrent pas dans d'autres programmes.

En somme, le processus se veut une démarche de concertation structurée. Au sujet de la démarche, un ADR explique que : "On ne voulait pas que le pacte rural soit considéré comme une enveloppe financière pour réaliser des projets. Il faut que ce soit une enveloppe financière pour réaliser des projets issus d'une démarche » (ADR, MRC 1). Et une autre, au sujet de la structuration de la démarche : « [cela implique de] faire une consultation publique et ensuite de développer un plan d'action et ensuite de développer des projets » (ADR, MRC 2).

\subsubsection{L'action des agents de développement rural}

Un constat du MAMROT sur les difficultés liées au travail en territoire dévitalisé a conduit à une augmentation du nombre d'ADR de la 1 re à la 2e PNR (de 104 à 136), notamment dans les MRC ayant le plus grand nombre de municipalités dévitalisées. Chaque MRC de cette étude compte deux ADR dont les rôles peuvent varier (municipalités ou MRC, concertation ou présence terrain, etc.), mais les fondements de leur action demeurent les mêmes. Les propos des informateurs font consensus quant à l'importance du rôle des ADR pour pallier le manque d'expertise dans les milieux plus défavorisés et apporter un soutien professionnel essentiel au développement. Les ADR ont pour rôle d'accompagner les municipalités dans leur développement: consultations publiques pour faire émerger les enjeux, les forces et la vision des citoyens; soutien au comité local de développement, à des groupes de bénévoles et au conseil municipal pour la production d'un plan d'action; animation de rencontres; gestion d'interfaces; etc. Au quotidien et en fonction des besoins du milieu, les ADR assurent la planification, la mise en 
œuvre, la gestion et le suivi des projets, tout en soutenant la recherche de financement, une fonction que les bénévoles ont souvent du mal à assumer. Plusieurs ADR s'impliquent dans d'autres politiques ou programmes (politique familiale, Municipalité amie des aînés) qui reposent également sur des consultations auprès des citoyens. Dans cet esprit, les ADR des trois MRC avaient des collaborations étroites avec les organisateurs communautaires du réseau de la santé et des services sociaux dans les démarches de caractérisation des communautés dont l'action a été présentée dans d'autres projets de recherche (Boisvert, 2007; Simard, Benazera et Charland, 2014). Dans les milieux très petits ou dévitalisés, la création, la consolidation ou le maillage d'entreprises privées, d'économie sociale ou de coopératives font partie des actions des ADR. Par leur connaissance approfondie des milieux, les ADR favorisent la collaboration autour de projets communs et relaient les préoccupations locales auprès de la MRC et autres organismes de la région, comme l'explique un préfet de MRC: «Les agentes rurales sont une source d'information évidemment fondamentale pour la MRC. Comme elles sont dans les milieux, elles peuvent nous amener les préoccupations, alimenter la réflexion de la MRC, elles ont en tête les plans de développement de leurs municipalités, les enjeux dans chaque municipalité. C'est un outil indispensable, les agents ruraux » (Préfet, MRC 1).

\subsubsection{Les projets collectifs déployés}

Dans chaque MRC il existe des projets territoriaux (pour toute la MRC), des projets inter-municipaux (quelques municipalités) et des projets locaux (une municipalité). Sur les 462 projets répertoriés entre 2007 et 2014 dans les trois MRC de la recherche, 105 sont des projets territoriaux ou intermunicipaux. Ils sont souvent reliés à la planification stratégique de la MRC et ils ne font pas l'objet d'une autre consultation. On y trouve deux constantes : l'accès à Internet haute-vitesse et l'amélioration du transport collectif. Deux MRC ont d'autres projets d'envergure : la création d'un site d'observation astronomique et le Parc régional des
Appalaches (Montmagny) et le projet d'une Maison de jeunes « Partenaires 12-18 » (Arthasbaska). Les autres projets sont locaux. Vu l'approche émergente retenue, la nature des quelques 210 projets locaux varie grandement d'une municipalité à l'autre et même au sein de chacune. Ils peuvent être regroupés comme suit : création ou réfection d'infrastructures tel que lieux de rencontre, activité physique, culture, services de proximité, attraction et rétention de résidents ou de travailleurs, aménagement d'espaces verts, animation festive, revitalisation d'école, création d'emplois, projets pour différents groupes d'âge. La plupart des municipalités ont également retenu un projet plus structurant (emploi, service de proximité essentiel, cohésion sociale, etc.), qu'il s'agisse d'un dépanneur, d'une résidence pour personnes âgées, d'une vocation particulière pour une école primaire ou d'un marché public. Le rôle des ADR a été déterminant pour la conception, le financement et le démarrage des projets. Toutefois, une fois ces étapes franchies, la pérennisation de nombreux projets repose sur la capacité d'agir des bénévoles. Certains projets ont généré des ressources ayant permis l'embauche de professionnels (résidence pour personnes âgées), mais le caractère durable de certains projets a pu être en péril considérant la charge de travail reposant sur les bénévoles.

\subsection{Objectif 2 : Identifier les déterminants sur lesquels les stratégies de la PNR prétendent agir et repérer les indicateurs quantitatifs et qualitatifs permettant de les documenter}

L'analyse des processus de mise en œuvre de la PNR (mécanismes de gouvernance des pactes ruraux, rôles des ADR et projets en cours) dans les trois MRC a permis d'identifier les principaux déterminants de la santé sur lesquels cette politique agit. La figure 2 présente les déterminants de la santé les plus influencés par la PNR, principalement au niveau des milieux de vie (la communauté locale et voisinage, ainsi que le milieu scolaire et de garde). 


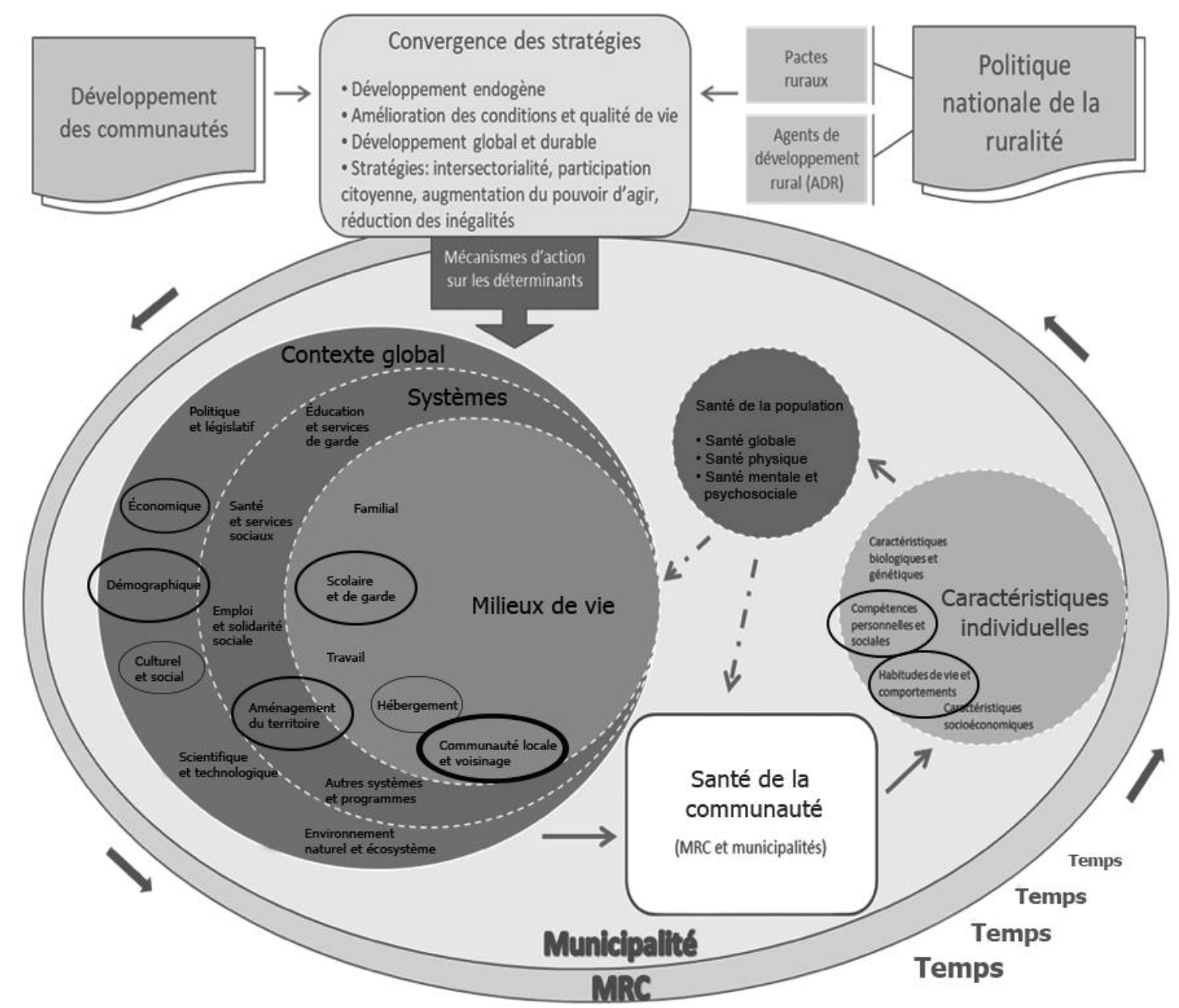

Figure 2-Les principaux déterminants de la santé touchés par la PNR

Un même projet peut avoir des retombées dans plusieurs champs et dimensions. Par exemple, la création d'une coopérative d'alimentation favorise de meilleures habitudes de vie par son offre de produits locaux et de mets préparés, renforce le tissu social et enrichit la vie communautaire par son emplacement et le fonctionnement coopératif dans lequel peuvent s'engager les individus. Une coopérative d'alimentation peut contribuer à influencer la démographie en rendant accessible des services ou en dynamisant des localités, ce qui peut attirer de nouveaux résidents (voir un autre exemple à la figure 4). De même, les processus à l'œuvre à travers ces projets renforcent les milieux de vie de multiples façons. Les personnes rencontrées ont souvent fait mention de la fierté vécue à travers la participation citoyenne, l'acquisition de compétences et les collaborations entre organismes. Outre l'impact de chaque projet, ce sont les processus animés par les ADR qui constitue, selon plusieurs, la plus grande réussite de la PNR. Un agent de développement note : «Moi je trouve qu'une des principales retombées c'est que je constate une augmentation du niveau de confiance des gens dans leur milieu à pouvoir prendre leur développement en main » (ADR, MRC 3). 


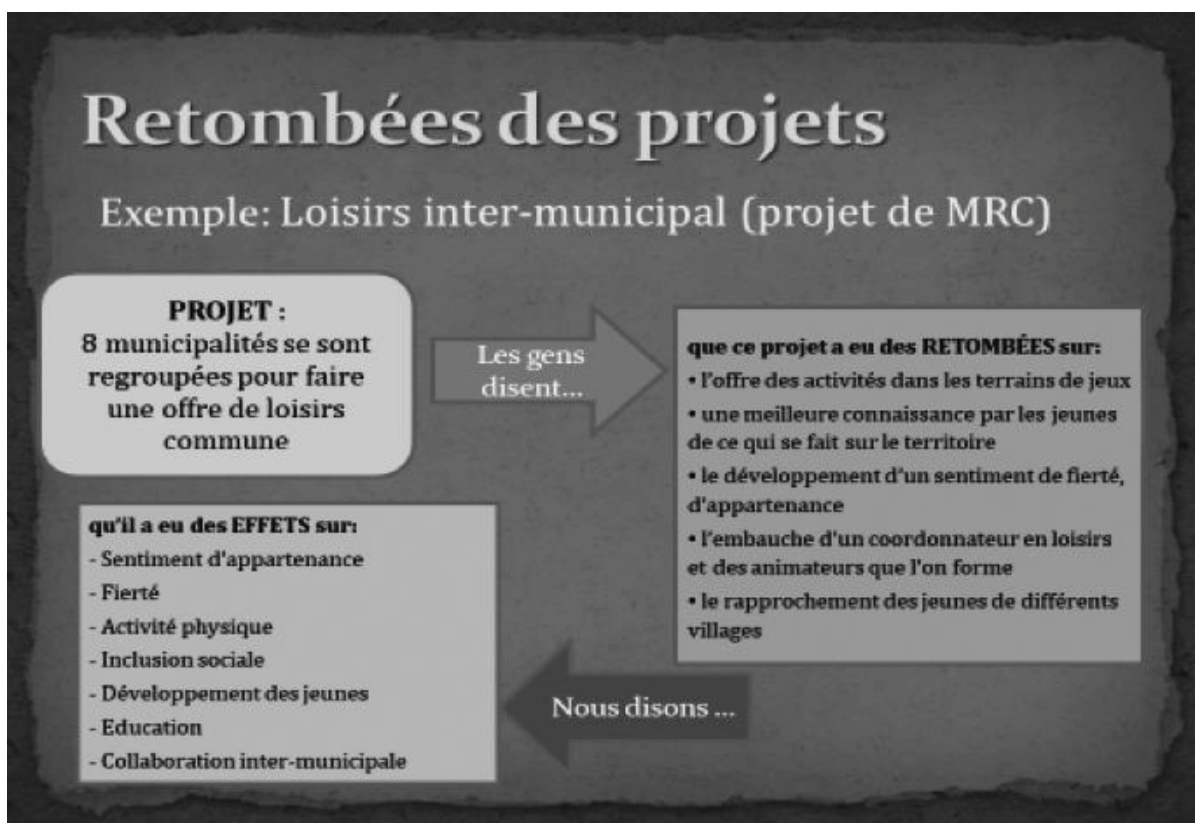

Figure 3 - Exemple : Le loisir intermunicipal (projet de MRC)

\subsection{Objectif $3:$ Mettre en lumière les effets de la PNR sur la santé et le bien-être des individus et des communautés}

Le cadre conceptuel retenu a permis de mettre en lumière les impacts des processus et des projets sur la santé des personnes et de la communauté. Les perceptions des personnes interviewées sur les retombées de la PNR ont été analysées sous deux angles : 1) en fonction des processus générés par les mécanismes de gouvernance; 2) en fonction de la nature des projets. Par exemple, la mobilisation des résidents pour mettre sur pied une salle d'entrainement physique peut renforcer les liens sociaux (processus) tout en créant un environnement favorable et en encourageant de meilleures habitudes de vie (projet). En effet, l'analyse révèle que les projets mis en place grâce aux ressources de la PNR (humaines et matérielles) peuvent agir sur les conditions de vie ou encourager de saines habitudes de vie, contribuant positivement à ces déterminants de la santé. Les processus de mise en place des projets peuvent créer une dynamique sociale positive, valorisant la participation citoyenne ou l'inclusion sociale, une dynamique associée à une meilleure santé globale, autant sur le plan physique que psychosocial, puis sur celle des individus. Cette étude confirme et illustre que les acteurs du réseau de la santé ne sont pas les seuls à avoir un impact sur la santé.

\section{Discussion}

Les résultats mettent en lumière la contribution des projets à fournir des services, à encourager les saines habitudes de vie ou à favoriser la cohésion sociale affectant positivement les déterminants de la santé. Les processus liés à l'émergence de ces projets permettent, quant à eux, aux membres de la communauté de se donner une vision de leur développement, d'agir sur la rétention de la population, de susciter un sentiment d'appartenance, de créer des collaborations ou de mobiliser la population sur divers aspects la concernant. Pourtant, même s'il a été possible de repérer des retombées de processus et de projets dans la plupart des champs de déterminants identifiés sur la Carte, certaines limites du cadre conceptuel sont apparues très tôt dans notre réflexion et ont été confirmées par nos analyses. Par exemple, la grande majorité des déterminants touchés par le processus des Pactes ruraux se sont retrouvés dans la catégorie « communauté locale et voisinage », dans le champ « milieux de vie ». Cette catégorie comprend toutes les dimensions de la vie en communauté, ce qui ne permet pas de rendre compte de la complexité des liens entre la dynamique d'une communauté, sa mise en mouvement et la santé globale des individus et de la communauté elle-même. De plus, lors de la recension des projets mis en place, une certaine « dérive vers les saines habitudes de vie » a été observée, dérive bien documentée dans la littérature de promotion de la 
santé, qui démontre la tendance des politiques à reconnaître la nécessité d'agir en amont sur les déterminants sociaux des inégalités de santé, mais de se concentrer largement sur les modes de vie individuels dans leur mise en œuvre (Shrecker et Taler, 2017). Si les projets recensés ont certes le potentiel d'agir sur les milieux de vie, leur impact sur les déterminants structuraux de la santé est limité, tout comme leur capacité à réduire les inégalités sociales de santé. De nouveau, le cadre conceptuel retenu n'a pas permis une analyse de l'interaction entre tous les déterminants.

Par ailleurs, bien qu'il soit juste d'avancer que des politiques novatrices telles que la PNR jouent vraisemblablement un rôle structurant pour les communautés territoriales, il est difficile de dissocier les effets de la PNR des actions menées par le biais d'autres politiques sociales (ex : politique familiale, Municipalités amies des aînés, etc.) ou des politiques macroéconomiques, celles nationales et internationales. Manifestement, la PNR n'a pu, à elle seule, contrer l'appauvrissement des territoires ruraux, déterminé par un contexte global qui dépasse la portée des territoires à l'étude. En outre, si la PNR permettait aux communautés d'acquérir plus de contrôle sur leur environnement local, sa capacité à influencer le contexte global est limitée. Pour intervenir sur ce contexte, les acteurs locaux devront donc déployer une diversité de stratégies qui appuieront leur action à différents niveaux : 1) soutenir les mouvements sociaux qui partagent des préoccupations semblables; 2) adopter une perspective globale de la communauté et favoriser les actions locales soutenues par des entrepreneurs sociaux et des organisations communautaires; 3) s'intéresser aux questions politiques régionales et nationales pour en débattre localement avec les citoyens (Langille, 2016).

Enfin, on ne peut passer outre le retrait de la PNR3 et le démantèlement des institutions partenaires de cette recherche à la toute fin de la collecte des données. Nous constatons que ce virage s'est soldé par une rupture avec le modèle de dialogue social et réduit désormais les possibilités d'innover. Le partenariat entre l'État et la société civile dans l'élaboration des politiques publiques est ainsi remis en cause et affecte l'organisation des territoires ruraux (Richard, Bourque et Lachapelle, 2017). Les conséquences de ces transformations posent désormais l'enjeu de la capacité des communautés rurales à atteindre les objectifs mis de l'avant par la PNR et affaiblissent l'émergence des projets et des processus qui les sous-tendaient, ce qui peut, dans la perspective explorée dans cette étude, avoir un impact négatif sur la santé (Joyal, Pouquay et El-Batal, 2005). Il est donc important de rappeler aux décideurs que la santé est produite non seulement par l'accès aux soins de santé et les comportements individuels en matière de santé, mais aussi par d'autres facteurs sociaux et économiques tels que la pauvreté, l'éducation et l'accès à une diversité de ressources économiques et sociales (Niederdeppe et al., 2008).

\section{Conclusion}

Le cadre conceptuel reposant sur les déterminants de la santé a permis d'illustrer comment la PNR contribue à la santé des individus et des communautés rurales. Comme la troisième édition de la PNR ne verra pas le jour, il appert que le réseau de la santé et des services sociaux perd là un allié important pour l'amélioration de la santé de la population. Au regard des déterminants qui ont été le plus influencés par la PNR, serait-il juste de penser qu'à plus long terme, l'abrogation de la PNR aura des effets délétères sur la santé des individus et des communautés? Compte tenu des changements en cours dans les orientations et dans la configuration des acteurs du développement en milieu rural, de nombreuses questions de recherche surgissent, à la fois pour faire avancer la connaissance et pour soutenir les acteurs engagés dans le développement de leurs communautés. Comment l'évolution du rôle des personnes élues influencera-t-elle le développement territorial et quelle place occuperont les citoyens dans ces gouvernances locales? Les nouveaux mécanismes de gestion du développement rural pourront-ils créer les conditions nécessaires à la poursuite d'une action efficace sur les déterminants sociaux de la santé des communautés? Quel pouvoir les communautés territoriales auront-elles sur les orientations nationales, sur les gouvernances qui orientent les politiques ayant un impact direct sur leurs conditions de vie? Autant de questions à garder en vue pour les années à venir. 


\section{NOTES}

1 Portée par le ministère des Affaires municipales et de l'Occupation du territoire (MAMOT), la PNR repose sur l'idée que "pour se réaliser comme société moderne, le Québec a besoin d'une ruralité forte et vivante lui permettant d'occuper son territoire de façon dynamique et durable, de mettre à profit ses ressources collectives et d'assurer des conditions de vie équitables à tous ses citoyens » (MAMR, 2006). Elle traduit une volonté d'assurer la pérennité des milieux ruraux et de l'identité rurale et sa vision est fondée sur la mise en valeur de l'organisation sociale et du mode de vie des communautés rurales. Enfin, elle vise à assurer le développement du milieu rural et l'occupation dynamique du territoire. La première Politique nationale de la ruralité est adoptée en décembre 2001, pour une période de cinq ans (2002-2007). La PNR se veut citoyenne, modulée et décentralisée. Cette politique est ensuite renouvelée pour la période 2007-2014, période au cours de laquelle elle sera évaluée par l'OCDE et donnera lieu à l'embauche d'un total de 136 agents ruraux et à la réalisation de milliers de projets territoriaux et locaux.

\section{RÉFÉRENCES}

Bernoux, J.F. (2004). L'évaluation participative au service du développement social. Paris, France : Dunod.

Boisvert, R. (2007). Les indicateurs de développement des communautés : Vers le déploiement d'un dispositif national de surveillance. Rapport général. Trois-Rivières, Québec : Agence de la santé et des services sociaux de la Mauricie et du Centre-du-Québec.

Clément, M., Ouellet, F., Coulombe, L., Côté, C. et Bélanger, L. (1995). Le partenariat de recherche. Éléments de définition et ancrage dans quelques études de cas. Service social, 44(2), 147-164.

Darbellay, F. et Paulsen, T. (2008). Le défi de l'inter et transdisciplinarité. Concepts, méthodes et pratiques innovantes dans l'enseignement et la recherche. Lausanne, Suisse : Presses polytechniques et universitaires romandes.

Desrosiers, L. (2014). Le Québec rural, mythes, légendes urbaines et réalité, présentation faite au colloque : La ruralité au Québec depuis les États généraux du monde rural (1991) : entre l'action et la recherche, bilan et perspectives, Montréal, Québec, ACFAS mai 2014.

Lincoln, S.Y., Lynham, S.A. et Guba, G.E. (2018). Paradigmatic controversies, contradictions, and emerging confluences. Dans Denzin, K.N., Lincoln, S.Y. (dir.), The SAGE Handbook of Qualitative Research (5 $5^{\text {th }}$ ed., p.108-150). Londres, Angleterre: Sage Publications.

Jean, B., Dionne, S. et Desrosiers, L. (2014). Comprendre le Québec rural. Chaire de recherche du Canada en développement rural, Université du Québec à Rimouski.

Joyal, A., Pouquay, F. et El-Batal, K. (2005). La politique québécoise de la ruralité : le développement local à l'épreuve. Géographie, Économie, Société, 7(2), 193-211.

Langille, D. (2016). Follow the money: How business and politics define our health. Dans Raphael, D (dir.), Social determinants of health (p.470-490). Toronto, Canada: Canadian Scholars Press.

Marmot, M. (2008). Closing the gap in a generation: health equity through action on the social determinants of health. The Lancet, 372(9650), 1661-1669.

Mikkonen, J. et Raphael, D. (2010). Social determinants of healtb: The Canadian Facts. Toronto, Canada: York University School of Health Policy and Management.

Ministère de la Santé et des Services sociaux (2012). La santé et ses déterminants. Mieux comprendre pour mieux agir. Québec, Ministère de la santé et des services sociaux.

Ministère des Affaires municipales, des Régions et de l'Occupation du territoire (2006). La Politique nationale de la ruralité 20072014. Québec, Québec : Ministère des Affaires municipales, des Régions et de l'Occupation du territoire.

Minkler, M. et Wallerstein, N. (2008). Community-Based Participatory Research for Health. From Process to Outcomes. San-Francisco, CA: Jossey-Bass.

Niederdeppe, J., Bu, Q.L., Borah, P., Kindig, D.A. et Robert, S.A. (2008). Message design strategies to raise public awareness of social determinants of health and population health disparities. Milbank Quarterly, 86, 481-513.

Organisation de coopération et de développement économiques (2010). Examens de l'OCDE des politiques rurales : Québec, Canada. Paris, France : Éditions OCDE.

Paillé, P. et Mucchielli, A. (2012). L'analyse qualitative en sciences bumaines et sociales (3e éd.), Paris, France : Armard Colin. 
Pampalon, R., Hamel, D. et Gamache, P. (2010). Health inequalities in urban and rural Canada: Comparing inequalities in survival according to an individual and area-based deprivation index. Health \& Place, 16, 416-420.

Raphael, D. (2016). Social determinants of health (3e éd.). Toronto, Canada: Canadian Scholar's Press.

Richard, J., Bourque, D. et Lachapelle, R. (2017). Impacts de la transformation des politiques publiques sur le développement des territoires. Gatineau, Québec: Chaire de recherche du Canada en organisation communautaire.

Shrecker, T. et Taler, V. (2017). How to think about social determinants of health: Revitalizing the agenda in Canada. Dans Bourgeault, I.L., Labonté, R., Packer, C., Runnels, V. (dir.), Population health in Canada (p. 100-111). Toronto: Canadian Scholars Press.

Simard, P. (2005). Perspectives pour une évaluation participative des Villes et Villages en santé. Québec, Québec : Institut national de Santé publique du Québec.

Simard, P., Benazera, C. et Charland, M. (2014). Le Dispositif participatif de caractérisation des communautés locales: évaluation d’implantation dans trois régions du Québec. Québec, Québec : Institut national de santé publique du Québec.

Woods, M. (2006). Redefining the "Rural Question": The New "Politics of the Rural" and Social Policy. Social Policy \& Administration, 40(6), 579-595. 